\title{
Density Functional Theory Study of the Multimode Jahn-Teller Problem in the Fullerene Anion
}

\author{
Harry Ramanantoanina, ${ }^{[\mathrm{a}]}$ Maja Gruden-Pavlovic, ${ }^{*[a, b]}$ Matija Zlatar, ${ }^{[\mathrm{c}]}$ and Claude Daul ${ }^{[\mathrm{a}]}$
}

The fullerene anion, $\mathrm{C}_{60}{ }^{-}$, within the $I_{h}$ point group, is a spherical molecule subject to the $\mathrm{T} \otimes \mathrm{h}$ Jahn-Teller (JT) distortion. The descent in symmetry goes to the three epikernel subgroups, namely $D_{5 d r} D_{3 d r}$ and $D_{2 h}$. The last one completely removes the electronic degeneracy, whereas $D_{5 d}$ and $D_{3 d}$ structures are subject to further JT distortion, leading to $C_{2 h}$ minimum energy structure. The multideterminantal density functional theory approach was applied to calculate the JT parameters for all seven different structures of lower symmetry. The multimode

\section{Introduction}

The buckminsterfullerene, $\mathrm{C}_{60}$, being the most spherical molecule synthesized to date, ${ }^{[1]}$ belongs to the icosahedral $\left(I_{h}\right)$ point group. Within this highest point group, the lowest unoccupied molecular orbital (LUMO) of $C_{60}$ is threefold degenerate, forming the bases for $T_{1 u}$ representation of $I_{h}$ group. The low electron affinity observed in $C_{60}{ }^{[2]}$ leads to the formation of a radical anion, ${ }^{[3]}$ as well as various polyanions. ${ }^{[4-8]}$ Much attention has been paid to the Jahn-Teller (JT) effect of fullerene ions in various electronic states as it plays an important role in the superconductivity mechanism of the alkali doped fullerides and has been a subject of interest of numerous groups. ${ }^{[9-13]}$

In the electronic structure of the icosahedral $\mathrm{C}_{60}^{-}$ion, one electron is occupying the LUMO $t_{1 u}$ orbitals. The resulting electronic state, ${ }^{2} T_{1 u r}$ is subject to the JT distortion. ${ }^{[14-17]} \mathrm{A}$ group theoretical analysis of the JT effect shows that in the threefold degenerate electronic state of the $I_{h}$ point group, the vibrations that belong to the $h_{g}$ irreducible representations (irreps) are the JT active $\left(T_{1 u} \otimes T_{1 u}=a_{g} \oplus h_{g}\right)$. Although the analysis of the interaction of $t_{1 u}$ orbital with a single $h_{g}$ vibrational mode is a good starting point to model the problem, by using simply group theory, is not possible to find the minimum energy conformation in which the distorted molecule is likely to be and how large the corresponding distortion and energy stabilization are. Moreover, complications in the analysis arise if several $h_{g}$ modes are responsible for the distortion. To address these issues, it is compulsory to carry out detailed computational study. Theoretical investigation of the JT effect in $C_{60}^{-}$ion, to our knowledge, were either constrained to the three epikernel subgroups of $I_{h}$ point group, namely $D_{5 d}, D_{3 d}$ and $D_{2 h}{ }^{[18-20]}$ or to the finding of the lowest energy distortion without any symmetry considerations. ${ }^{[21]}$ Furthermore, experimentally observed distorted structure of fullerene anion may appear in different low symmetry (LS) point groups depending on the environment. ${ }^{[22]}$ In this study, the results obtained by problem in this system was addressed using the intrinsic distortion path method, in which the JT distortion is expressed as a linear combination of all totally symmetric normal modes in the particular low symmetry minimum energy conformation. Results obtained by both methods are consistent and give direct insight into the coupling of electronic distribution and nuclear movements in $\mathrm{C}_{60}^{-}$.

multideterminantal density functional theory (MDDFT ${ }^{[23-28]}$ for the distortion of $\mathrm{C}_{60}^{-}$ion, for the epikernel subgroups of the $I_{h}$ point group as well as their further distortion to $C_{2 h}$ is presented. To tackle the multimode problem, the intrinsic distortion path (IDP) analysis ${ }^{[23,24 b]}$ in which the distortion is represented as a superposition of all totally symmetric normal modes in the LS point groups, has been performed. The IDP analysis answers the questions, which totally symmetric normal coordinates of the LS structure contribute to the JT distortion at the high symmetry (HS) point, and how does these contributions change along the minimal energy path toward the particular distorted energy minimum. Thus, in this work, we will show how density functional theory (DFT)-based models are able, not only to quantify the JT effect, but also to get deep insight of its microscopic origin and hence to understand the electronic structure of this molecule. It is noteworthy that our model is completely theoretical and without fitting of parameters to the experimental data.

\section{Methodology}

\section{The multideterminantal DFT approach}

The method for the calculation of the JT parameters, within the DFT framework, has been successfully applied for the

[a] H. Ramanantoanina, M. Gruden-Pavlovic, C. Daul Department of Chemistry, University of Fribourg, Chemin du Musée 9, $\mathrm{CH}-1700$ Fribourg, Switzerland

E-mail: gmaja@chem.bg.ac.rs

[b] M. Gruden-Pavlovic

Department of General and Inorganic Chemistry, Faculty of Chemistry, University of Belgrade, Studentski trg 12-16, 11000 Belgrade, Serbia

[c] M. Zlatar

Center for Chemistry, Institute of Chemistry, Technology and Metallurgy,

University of Belgrade, Studentski trg 12-16, 11000 Belgrade, Serbia

Contract grant sponsor: Swiss National Science Foundation, the Serbian Ministry of Education and Science; contract grant number: 172035.

(C) 2012 Wiley Periodicals, Inc. 


\begin{tabular}{|c|c|c|c|c|}
\hline Distortion & $\Gamma_{\mathrm{el}}{ }^{[\mathrm{a}]}$ & $\Gamma_{J T^{[b]}}^{[b]}$ & $N_{\mathrm{a} 1}{ }^{[c]}$ & $\begin{array}{l}\text { Origin of the } \\
\text { LS } a_{1} \text { vibration }\end{array}$ \\
\hline$I_{h} \rightarrow D_{5 d}$ & $T_{1 u} \rightarrow A_{2 u}+E_{1 u}$ & $h_{g} \rightarrow a_{1 g}+e_{1 g}+e_{2 g}$ & 10 & $2 a_{g}, 8 h_{g}$ \\
\hline$I_{h} \rightarrow D_{3 d}$ & $T_{1 u} \rightarrow A_{2 u}+E_{u}$ & $h_{g} \rightarrow a_{1 g}+2 e_{g}$ & 16 & $2 a_{g}, 6 g_{g}, 8 h_{g}$ \\
\hline$I_{h} \rightarrow D_{2 h}$ & $T_{1 u} \rightarrow B_{1 u}+B_{2 u}+B_{3 u}$ & $h_{g} \rightarrow 2 a_{g}+b_{1 g}+b_{2 g}+b_{3 g}$ & 24 & $2 a_{g}, 6 g_{g}, 8 h_{g}$ \\
\hline$I_{h} \rightarrow C_{2 h}$ & $T_{1 u} \rightarrow A_{u}+2 B_{u}$ & $h_{g} \rightarrow 3 a_{g}+2 b_{g}$ & 45 & $2 a_{g}, 3 \mathrm{t}_{1 g}, 4 \mathrm{t}_{2 g}, 6 g_{g}, 8 h_{g}$ \\
\hline
\end{tabular}

[a] $\Gamma_{\mathrm{el}}$ irrep of the electronic state. [b] $\Gamma_{\text {JT }}$ the irrep of the JT active vibrations. [c] $N_{\mathrm{a} 1}$ the number of totally symmetrical vibrations in the LS point group.

analysis of JT active molecules. ${ }^{[23-29]}$ In short, it is necessary to know the geometries and the energies of the HS and LS nuclear configuration. For the case of the LS conformation, since the system is in a nondegenerate electronic ground state, the geometry, as well as the corresponding energy, is obtained by standard DFT, whereas for the HS conformation the electronic state must be represented by more than one Slater Determinant, in this case by three. Alternately, one can use some constrained electron configuration method. ${ }^{[27]}$ The geometry of the HS structure is obtained using the so-called Average of Configuration approach ${ }^{[23]}$ i.e., by taking the subspace, density for the three states of the $t$ irreps and dividing by the dimension of the subspace which in this case is three. ${ }^{[30]}$ In this way, the density has the symmetry of the external potential, i.e., it belongs to the totally symmetric irrep of the point group under which the Hamiltonian is invariant. The energy of the HS structure is calculated imposing obtained HS geometry and the LS electron density, by adequate occupation of MO's in proper LS point group. The JT stabilization energy $\left(E_{J T}\right)$ is obtained by the difference between the energies corresponding to the HS and LS geometries with the same electron distribution. The warping barrier $(\Delta)$ is given by the difference in energies between the LS structures with different electronic states arising from the splitting of the originally degenerate electronic state in the HS point group. The JT radius $\left(R_{J T}\right)$ is obtained as the norm of the distortion vector between the HS and LS geometries.

The DFT calculations have been carried out using the Amsterdam Density Functional (ADF) Program Package, version ADF2009.01. ${ }^{[31]}$ The local density approximation characterized by the Vosko-Willk-Nussair ${ }^{[32]}$ parameterization have been used for the symmetry constrained geometry optimization, single point calculations, and analytical frequency calculations. The carbon atom was represented by triple-zeta slater-type orbitals plus one polarization function basis set, and all the calculations were spin-unrestricted.

\section{The IDP}

The concept of the IDP for the analysis of the JT effect is based on the fact that all the information about the vibronic coupling at the HS nuclear arrangement is also contained in the distorted energy minimum structure. Hence, the distortion can be given as a superposition of all totally symmetric normal modes in the LS point group. The IDP is defined as the minimal energy path of the potential energy surface, connecting the HS configuration with each LS structure. It is noteworthy, that in the IDP model, not only first order JT active modes (in the present case $h_{g}$ modes) are considered, but also all the other modes that become totally symmetric upon descent in symmetry (Table 1). The choice of the LS geometry as the reference point is in contrast to the usual treatment of the JT effect. This point corresponds to an energy minimum and has the property that the Hessian of the energy is positive semi-definite and thus can be used to obtain the harmonic vibrational modes without any complications. Details about this approach can be found in Refs. [23c, 24, and 33].

\section{Results and Discussion}

The $\mathrm{C}_{60}^{-}$ion has $3 \mathrm{~N}-6=174$ normal modes, which can be classified according to the irreps of the $I_{h}$ point group, $2 a_{g} \oplus 3 t_{1 g}$ $\oplus 4 t_{2 g} \oplus 6 g_{g} \oplus 8 h_{g} \oplus a_{u} \oplus 4 t_{1 u} \oplus 5 t_{2 u} \oplus 6 g_{u} \oplus 7 h_{u}$, while the electronic ground state is ${ }^{2} T_{1 u}$. Hence, this anion is prone to the vibronic coupling of the triply degenerate electronic state with the fivefold degenerate nuclear motion, leading to the $T \otimes h$ JT problem ${ }^{[15,16]}$ (Table 1). According to the Epikernel principle, ${ }^{[18]}$ three general distortion pathways from the $I_{h}$ structure may be highlighted: the descent in symmetry to $D_{5 d}$ (a), $D_{3 d}$ (b), and $D_{2 h}$ (c) point groups. The latter one removes completely the electronic degeneracy. However, in $D_{5 d}$ and $D_{3 d}$ point groups, one component of the electronic state remains degenerate, leading to the typical $E \otimes e$ JT problem, ${ }^{[15,16]}$ with a descent in symmetry to $C_{2 h}$ (Fig. 1). Consequently, seven unique LS geometries, resulting from four possible distortion pathways, are considered in the determination of the JT parameters in the $\mathrm{C}_{60}^{-}$ion.

The MDDFT calculations of the JT effect in $\mathrm{C}_{60}^{-}$ion reveal a small distortion of the geometry, as previously predicted with other theoretical ${ }^{[34]}$ and experimental ${ }^{[35]}$ studies. In all cases, the $E_{\mathrm{JT}} \mathrm{S}$ obtained from IDP are consistent with the MDDFT calculations (312 $\mathrm{cm}^{-1}$, Tables $\left.2-4\right)$. These results agree with previous DFT studies, ${ }^{[36,37]}$ which report $E_{\text {JT }}$ between 300 and 640 $\mathrm{cm}^{-1}$, depending on the functional used, where hybrid functionals, not surprisingly, give somewhat larger values. The estimated $E_{\text {JT }}$ from visible and near-infrared spectra ${ }^{[38]}$ is 386 and $467 \mathrm{~cm}^{-1},{ }^{[12]}$ while data derived from the photoemission spectra of Gunnarsson et al. ${ }^{[13]}$ gave the value of $848 \mathrm{~cm}^{-1}$. More recent reinterpretation of the same spectra resulted in the value of $754 \mathrm{~cm}^{-1},{ }^{[9,36]}$ and $E_{\mathrm{JT}}$ energy derived from photoelectron spectrum measurements of Wang ${ }^{[9]}$ is 594 or $409 \mathrm{~cm}^{-1}$, depending on the simulation method. ${ }^{[36]}$ The values obtained by CASSCF calculations ${ }^{[36]}$ are two times larger than the experimental ones. Summary of various experimental and theoretical results on $\mathrm{C}_{60}^{-}$is recently published. ${ }^{[36]}$ Hence, quantifying the JT distortion using the MDDFT method is simple and efficient and is valuable, since experimental determination of the 


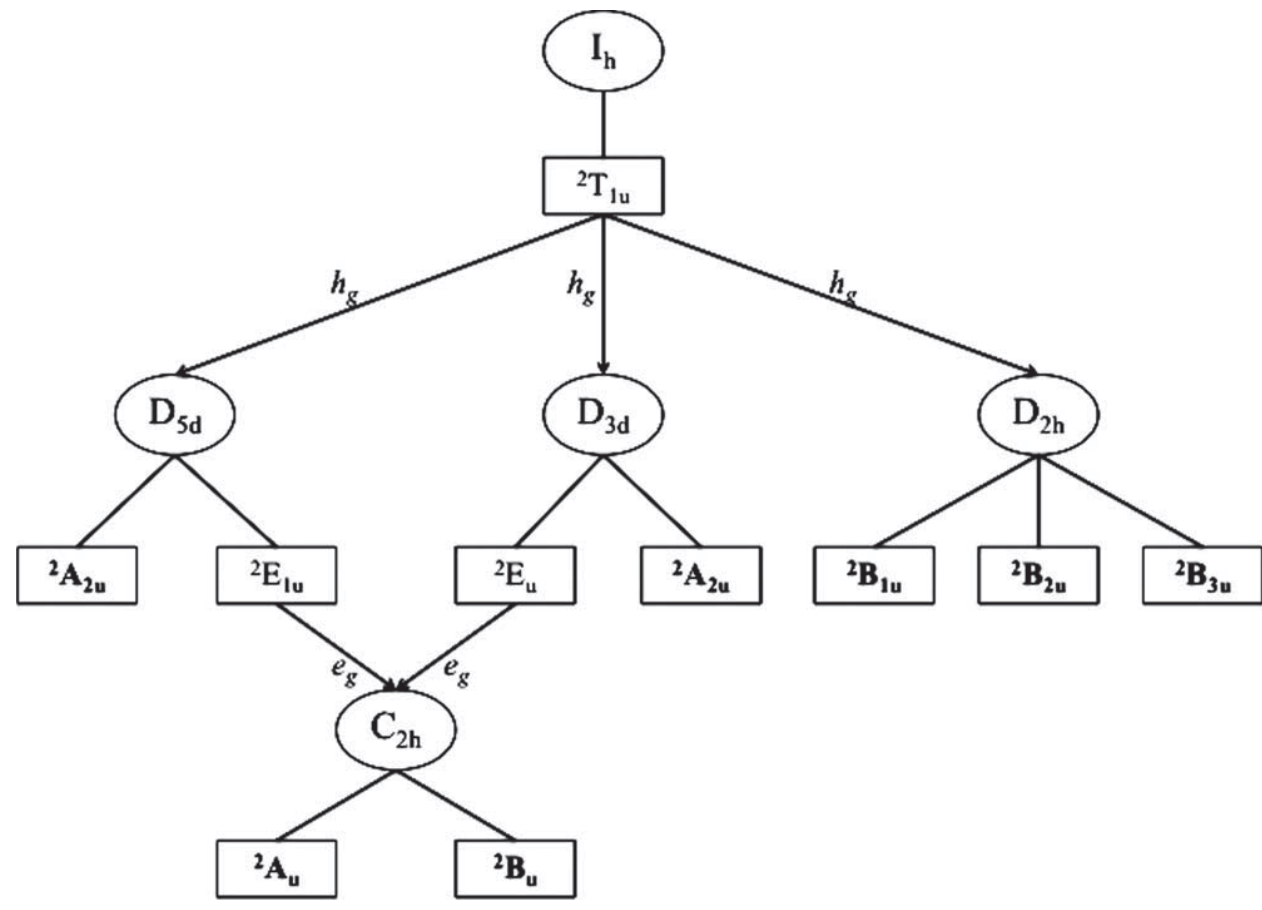

Figure 1. Part of the descent in symmetry chain of the $I_{h}$ point group. Subgroups, electronic states and the symmetry of the vibrations are highlighted.

JT parameters is very difficult and there is no consistency in the values obtained by the different methods.

The results corresponding to the $I_{h} \rightarrow D_{5 d} \rightarrow C_{2 h}$ (Table 2), $I_{h} \rightarrow D_{3 d} \rightarrow C_{2 h}$ (Table 3) and $I_{h} \rightarrow D_{2 h}$ (Table 4) obtained by both MDFT and IDP, show that the energy differences between the different states on the potential energy surfaces are small (the maximum energy differences is about $15 \mathrm{~cm}^{-1}$ ). According to the MDDFT calculations, the ${ }^{2} A_{u}\left(C_{2 h}\right)$ state is a global minimum on the potential energy surface, regardless of the distortion path.

Table 2. Results of the DFT calculations and IDP method corresponding to the descent in symmetry from $I_{\mathrm{h}}$ to $D_{5 d}$ and further to $C_{2 \mathrm{~h}}$.

\begin{tabular}{|c|c|c|c|}
\hline Occupation & $\begin{array}{l}\text { Electronic } \\
\text { state }\end{array}$ & $\begin{array}{l}\text { Nuclear } \\
\text { geometry }\end{array}$ & Energy $^{[a]}$ \\
\hline$t_{11}{ }^{1 / 3} t_{1 u^{1 / 3}} t_{11}{ }^{1 / 3}$ & ${ }^{2} T_{1 u}$ & $I_{h}$ & -583.0921 \\
\hline$a_{2 u} e_{1 u^{0}} e_{1 u^{0}}$ & ${ }^{2} A_{2 u}$ & $I_{h}$ & -583.0826 \\
\hline$a_{2 u}{ }^{0} e_{1 u^{1 / 2}} e_{1 u^{1 / 2}}$ & ${ }^{2} E_{1 u}$ & $I_{h}$ & -583.0897 \\
\hline$b_{u}{ }^{0} a_{u}{ }^{1} b_{u}{ }^{0}$ & ${ }^{2} A_{u}$ & $I_{h}$ & -583.0825 \\
\hline$b_{u}^{0} a_{u}{ }^{0} b_{u}{ }^{1}$ & ${ }^{2} B_{u}$ & $I_{h}$ & -583.0825 \\
\hline$a_{2 u}{ }^{1} e_{1 u}{ }^{0} e_{1 u}{ }^{0}$ & ${ }^{2} A_{2 u}$ & $D_{5 d}$ & -583.1194 \\
\hline$b_{u}{ }^{0} a_{u}{ }^{1} b_{u}{ }^{0}$ & ${ }^{2} A_{u}$ & $C_{2 h}$ & -583.1211 \\
\hline$b_{u}^{0} a_{u}^{0} b_{u}{ }^{1}$ & ${ }^{2} B_{u}$ & $C_{2 h}$ & -583.1210 \\
\hline$E_{\mathrm{JT}}$ & ${ }^{2} A_{2 u}$ & $I_{h} \rightarrow D_{5 d}$ & 297 \\
\hline$E_{\mathrm{JT}}$ & ${ }^{2} A_{u}$ & $I_{h} \rightarrow C_{2 h}$ & 312 \\
\hline$E_{\mathrm{JT}}$ & ${ }^{2} B_{u}$ & $I_{h} \rightarrow C_{2 h}$ & 312 \\
\hline$R_{\mathrm{JT}}{ }^{[\mathrm{b}]}$ & ${ }^{2} A_{2 u}$ & $I_{h} \rightarrow D_{5 d}$ & $6.4110^{-2}$ \\
\hline$R_{\mathrm{JT}}{ }^{[\mathrm{b}]}$ & ${ }^{2} A_{u}$ & $I_{h} \rightarrow C_{2 h}$ & $6.4610^{-2}$ \\
\hline$R_{\mathrm{JT}}{ }^{[\mathrm{b}]}$ & ${ }^{2} B_{u}$ & $I_{h} \rightarrow C_{2 h}$ & $6.5110^{-2}$ \\
\hline$E_{\mathrm{JT}}(\mathrm{IDP})$ & ${ }^{2} A_{2 u}$ & $I_{h} \rightarrow D_{5 d}$ & 305 \\
\hline$E_{\mathrm{JT}}(\mathrm{IDP})$ & ${ }^{2} A_{u}$ & $I_{h} \rightarrow C_{2 h}$ & 302 \\
\hline$E_{\mathrm{JT}}(\mathrm{IDP})$ & ${ }^{2} B_{u}$ & $I_{h} \rightarrow C_{2 h}$ & 302 \\
\hline
\end{tabular}

[a] Energies are given in eV; $E_{\mathrm{JT}}$ is given in $\mathrm{cm}^{-1}$. [b] $R_{\mathrm{JT}}$ in $\AA$.
From group theoretical considerations, ${ }^{[19]}$ it is expected that the nuclear arrangements belonging to $D_{5 d}$ and $D_{3 d}$ point groups could be either maximum or minimum on the potential energy surface, while the $D_{2 h}$ geometry is a saddle point on the potential energy surface. The DFT calculations do not reveal these distinctions as small energy changes between different distorted structures fall in the range of the precision of the calculations. However, it should be pointed out that even if the energy differences are small, the $C_{2 h}$ structure is the global minimum on the potential energy surface, although it is usually not considered in the analysis of the linear JT effect in $\mathrm{C}_{60}^{-}$ion, neither group theoretical nor computational. No warping of the potential energy surface is observed in the $D_{2 h}$ pathway and the LS geometries with the ${ }^{2} B_{1 u r}{ }^{2} B_{2 u r}$ and ${ }^{2} B_{3 u}$ electronic states are isoenergetic (Table 4).

The distortion generally occurs along the axis that becomes principal axis in the LS point group (i.e, elongation along the $C_{5}$ axis in the $D_{5 d}, C_{3}$ axis in the $D_{3 d}$ and $C_{2}$ axis in the $D_{2 h}$ structures). In such a situation, the distortion is represented as a prolate deformation of the spherical $C_{60}$ cage along the polar axes. Otherwise, the distortion corresponds to an oblate deformation of the spherical $C_{60}$ cage. The minimum energy

Table 3. Results of the DFT calculations and IDP method corresponding to the descent in symmetry from $I_{h}$ to $D_{3 d}$ and further to $C_{2 h}$.

\begin{tabular}{|c|c|c|c|}
\hline Occupation & $\begin{array}{c}\text { Electronic } \\
\text { state }\end{array}$ & $\begin{array}{l}\text { Nuclear } \\
\text { geometry }\end{array}$ & Energy $^{[a]}$ \\
\hline$t_{1 u}{ }^{1 / 3} t_{1 u^{1 / 3}} t_{1 u^{1 / 3}}$ & ${ }^{2} T_{1 u}$ & $I_{h}$ & -583.0921 \\
\hline$a_{2 u}{ }^{1} e_{u}{ }^{0} e_{u}{ }^{0}$ & ${ }^{2} A_{2 u}$ & $I_{h}$ & -583.0826 \\
\hline$a_{2 u}{ }^{0} e_{u}{ }^{1 / 2} e_{u}{ }^{1 / 2}$ & ${ }^{2} E_{u}$ & $I_{h}$ & -583.0900 \\
\hline$b_{u}^{0} a_{u}{ }^{1} b_{u}{ }^{0}$ & ${ }^{2} A_{u}$ & $I_{h}$ & -583.0825 \\
\hline$b_{u}^{0} a_{u}^{0} b_{u}{ }^{1}$ & ${ }^{2} B_{u}$ & $I_{h}$ & -583.0825 \\
\hline$a_{2 u}{ }^{1} e_{u}^{0} e_{u}^{0}$ & ${ }^{2} A_{2 u}$ & $D_{3 d}$ & -583.1204 \\
\hline$b_{u}^{0} a_{u}{ }^{1} b_{u}^{0}$ & ${ }^{2} A_{u}$ & $C_{2 h}$ & -583.1210 \\
\hline$b_{u}^{0} a_{u}^{0} b_{u}{ }^{1}$ & ${ }^{2} B_{u}$ & $C_{2 h}$ & -583.1209 \\
\hline$E_{\mathrm{JT}}$ & ${ }^{2} A_{2 u}$ & $I_{h} \rightarrow D_{3 d}$ & 305 \\
\hline$E_{\mathrm{JT}}$ & ${ }^{2} A_{u}$ & $I_{h} \rightarrow C_{2 h}$ & 312 \\
\hline$E_{\mathrm{JT}}$ & ${ }^{2} B_{u}$ & $I_{h} \rightarrow C_{2 h}$ & 312 \\
\hline$R_{\mathrm{JT}}{ }^{[\mathrm{b}]}$ & ${ }^{2} A_{2 u}$ & $I_{h} \rightarrow D_{3 d}$ & $6.6310^{-2}$ \\
\hline$R_{\mathrm{JT}}{ }^{[\mathrm{b}]}$ & ${ }^{2} A_{u}$ & $I_{h} \rightarrow C_{2 h}$ & $6.5510^{-2}$ \\
\hline$R_{\mathrm{JT}}{ }^{[\mathrm{b}]}$ & ${ }^{2} B_{u}$ & $I_{h} \rightarrow C_{2 h}$ & $6.5010^{-2}$ \\
\hline$E_{\mathrm{JT}}(\mathrm{IDP})$ & ${ }^{2} A_{2 u}$ & $I_{h} \rightarrow D_{3 d}$ & 308 \\
\hline$E_{\mathrm{JT}}(\mathrm{IDP})$ & ${ }^{2} A_{u}$ & $I_{h} \rightarrow C_{2 h}$ & 305 \\
\hline$E_{\mathrm{JT}}(\mathrm{IDP})$ & ${ }^{2} B_{u}$ & $I_{h} \rightarrow C_{2 h}$ & 303 \\
\hline
\end{tabular}




\begin{tabular}{|c|c|c|c|}
\hline Occupation & $\begin{array}{l}\text { Electronic } \\
\text { state }\end{array}$ & $\begin{array}{l}\text { Nuclear } \\
\text { geometry }\end{array}$ & Energy $^{[\mathrm{a}]}$ \\
\hline$t_{1 u}{ }^{1 / 3} t_{1 u^{1 / 3}} t_{1 u}{ }^{1 / 3}$ & ${ }^{2} T_{1 u}$ & $I_{h}$ & -583.0921 \\
\hline$b_{1 u}{ }^{1} b_{2 u^{a}} b_{3 u}{ }^{0}$ & ${ }^{2} B_{1 u}$ & $I_{h}$ & -583.0826 \\
\hline$b_{1 u^{0}} b_{2 u^{1}} b_{3 u^{0}}$ & ${ }^{2} B_{2 u}$ & $I_{h}$ & -583.0826 \\
\hline$b_{1 u^{\circ}} b_{2 u^{0}} b_{3 u^{1}}$ & ${ }^{2} B_{3 u}$ & $I_{h}$ & -583.0826 \\
\hline$b_{1 u^{1}} b_{2 u^{0} b_{3 u^{0}}}$ & ${ }^{2} B_{1 u}$ & $D_{2 h}$ & -583.1208 \\
\hline$b_{1 u^{0}} b_{2 u^{1}} b_{3 u^{0}}$ & ${ }^{2} B_{2 u}$ & $D_{2 h}$ & -583.1208 \\
\hline$b_{1 u^{0}} b_{2 u^{1}} b_{3 u^{0}}$ & ${ }^{2} B_{3 u}$ & $D_{2 h}$ & -583.1209 \\
\hline$E_{\mathrm{JT}}$ & ${ }^{2} B_{1 u}$ & $I_{h} \rightarrow D_{2 h}$ & 305 \\
\hline$E_{\mathrm{JT}}$ & ${ }^{2} B_{2 u}$ & $I_{h} \rightarrow D_{2 h}$ & 310 \\
\hline$E_{\mathrm{JT}}$ & ${ }^{2} B_{3 u}$ & $I_{h} \rightarrow D_{2 h}$ & 307 \\
\hline$R_{\mathrm{JT}_{\mathrm{T}}}^{[\mathrm{b}]}$ & ${ }^{2} B_{1 u}$ & $I_{h} \rightarrow D_{2 h}$ & $6.4010^{-2}$ \\
\hline$R_{\mathrm{JT}_{\mathrm{T}}}^{[\mathrm{bb}]}$ & ${ }^{2} B_{2 u}$ & $I_{h} \rightarrow D_{2 h}$ & $6.3010^{-2}$ \\
\hline$R_{J_{\top}}^{[b]}$ & ${ }^{2} B_{3 u}$ & $I_{h} \rightarrow D_{2 h}$ & $6.3210^{-2}$ \\
\hline$E_{\text {JT }}($ IDP) & ${ }^{2} B_{1 u}$ & $I_{h} \rightarrow D_{2 h}$ & 303 \\
\hline$E_{\mathrm{JT}}(\mathrm{IDP})$ & ${ }^{2} B_{2 u}$ & $I_{h} \rightarrow D_{2 h}$ & 298 \\
\hline$E_{\mathrm{JT}}(\mathrm{IDP})$ & ${ }^{2} B_{3 u}$ & $I_{h} \rightarrow D_{2 h}$ & 301 \\
\hline
\end{tabular}

[a] Energies are given in eV; $E_{\mathrm{JT}}$ is given in $\mathrm{cm}^{-1}$. [b] $R_{\mathrm{JT}}$ in $\AA$.

$C_{2 h}$ structures are consequence of such a distortion. Figure 2 represents the $D_{5 d}, D_{3 d}$ and $D_{2 h}$ distortion product by the different components of the lowest frequency $I_{h}$ squashing $h_{g}$ mode \#1, with the displacement along a fivefold, threefold, and twofold axes, respectively. A convenient way to represent such a vibration is to consider the nuclear displacement as a product of a distortion of the spherical surface proportional to the $\left(3 z^{2}-r^{2}\right)$ distortion along the fivefold axis (Fig. 2a). Then, the $D_{3 d}$ (Fig. 2b) and the $D_{2 h}$ (Fig. 2c) distortions are the consequences of a pseudo-rotation of the $D_{5 d}$ one.

The vibronic coupling can be quantified within the IDP method. Although a different number of totally symmetric modes is considered in the analysis of the multimode JT effect within the four different distortion paths $\left(I_{h} \rightarrow D_{5 d}\right.$, Fig. 3a; $I_{h} \rightarrow D_{3 d}$, Fig. 3b; $I_{h} \rightarrow D_{2 d}$, Fig. 3c; $I_{h} \rightarrow C_{2 h}$, Figs. 3d and $3 e)$, the same qualitative picture is obtained. One can distinguish two regions (Fig. 3a1, Fig. 3b1, and Fig. $3 c 1$ ) on the potential energy surface. In the first region the energy changes faster, while in the second region the geometry just relaxes towards the global minimum. The analysis of the multimode JT distortion shows that, regardless of the number of totally symmetric normal modes in the LS point group (Table 1), the energy stabilization is mostly achieved by the hardest frequency modes, while the relaxation of the geometry is encountered by softer frequency ones. In Figure 3(2), bold lines correspond to the vibrations, which contribute the most to the $R_{\mathrm{JT}}$ and $E_{\mathrm{JT}}$. Within the framework of the IDP model, the total contributions of all totally symmetric modes in the LS point group to the $R_{\mathrm{JT}}$ and $E_{\mathrm{JT}}$ have been calculated and are available in Supplementary Information (Supporting Information Tables S1, S2, S3, S4). All these modes can be correlated with the corresponding vibrations in the HS point group $^{[39]}$ thus having connection with the usual treatment of the JT effect. As it is predicted by group theoretic considerations (Table 1), vibrations that correlate to the $h_{g}$ modes in $I_{h}$ point group are responsible for the distortion, which indicates that no other nuclear movements are JT active. Out of the eight $h_{g}$ modes in $I_{h}$ point group, four modes stand out as being of crucial importance: the squashing deformations $h_{g} \# 1$ and $h_{g} \# 2$ are mostly responsible of the JT distortion of the geometry; the antisquashing deformations $h_{g} \# 7$ and $h_{g}$ \#8, which represent actually a C-C stretch vibration, are mostly responsible for the energy stabilization. Contributions of $h_{g}$ modes are in agreement with the multimode analysis of Iwahara et al., ${ }^{[9,36]}$ and Manini et al., ${ }^{[37]}$ although our analysis did not reveal significant contribution of $a_{g} \# 1$ and $a_{g} \# 2$ modes. This is probably due to the models employed in the multimode analysis, as well as due to the choice of the $I_{h}$ geometry. However, our results completely correspond to the recent analysis of the multimode JT distortion in $\mathrm{C}_{5} \mathrm{H}_{5}$. and $\mathrm{C}_{6} \mathrm{H}_{6}{ }^{+}$, which can be considered as the building blocks of fullerenes. ${ }^{[33]}$ The analysis of the $(\mathrm{E} \otimes \mathrm{e})$ problem, which leads from either $D_{5 d}$ or $D_{3 d}$ structures to $C_{2 h}$, gives the same qualitative picture.

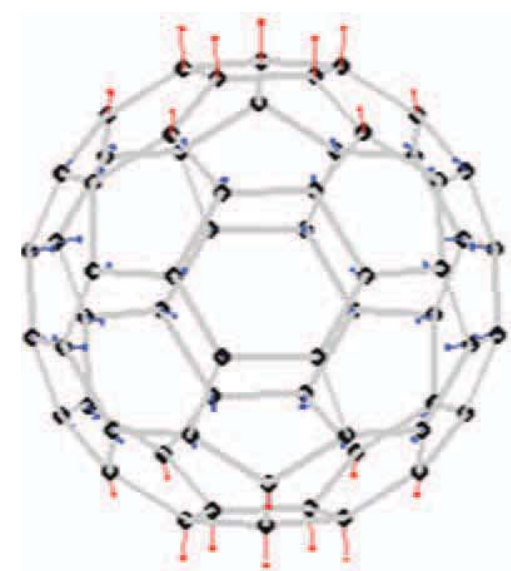

(a)

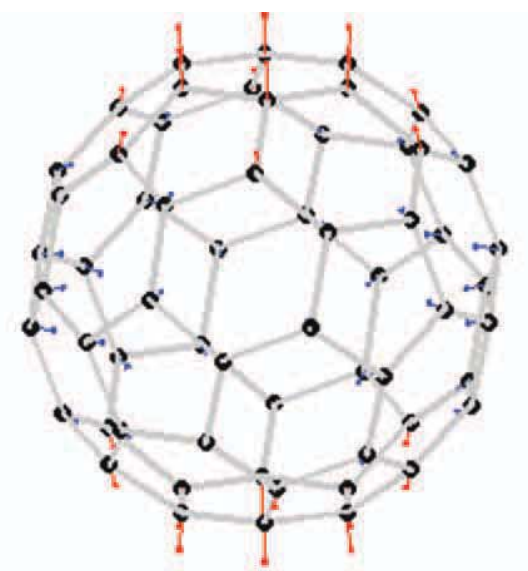

(b)

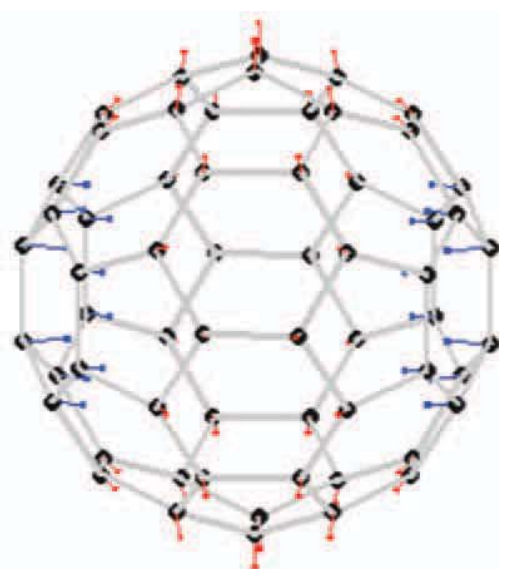

(c)

Figure 2. Graphical representations of the three components of $h_{\mathrm{g}} \# 1$ vibrations that yield to $D_{5 d}$ a), $D_{3 d}$ b), and $D_{2 h}$ c) structures. Red arrows indicate the displacements outwards the sphere and blue arrows indicate the displacement inwards the surface of the sphere. [Color figure can be viewed in the online issue, which is available at wileyonlinelibrary.com.] 

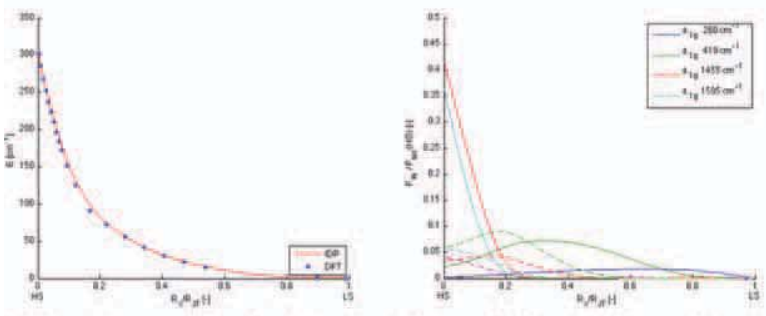

(1) Changes of the energy, IDP model and DFT results

(a) IDP of the $\pi \mathrm{l}_{\mathrm{h}}$ to $\mathrm{D}_{\mathrm{gd}}$ distortion in $\mathrm{C}_{\mathrm{eg}}$ ?
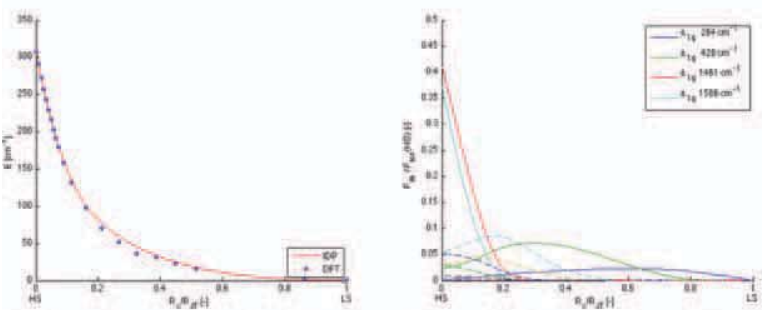

$\begin{array}{ll}\text { (1) Changes of the energy, IDP model and DFT results } & \text { (2) Changes of the forces of the } 16 \mathrm{a}_{\mathrm{t}} \text { modes along the }\end{array}$ (b) IDP of the $\int \mathrm{I}_{\mathrm{k}}$ to $\mathrm{D}_{34}$ distortion in $\mathrm{C}_{\mathrm{ga}}$.

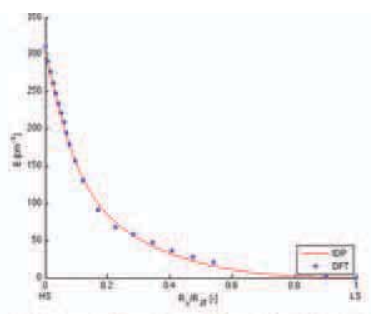

(1) Changes of the energy, IDP model and DFT results

(c) IDP of the JT $\mathrm{I}_{4}$ to $\mathrm{D}_{\text {sh }}$ distortion in $\mathrm{C}_{\mathrm{es}}$ :

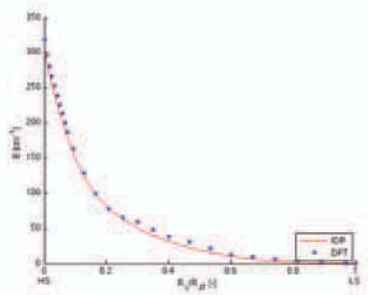

(1) Changes of the energy, IDP model and DFT results

(d) IDP of the $\mathrm{JT}_{4}$ to $\mathrm{C}_{2 h}$ ( ${ }^{2} \mathrm{~A}$ ) distortion in $\mathrm{C}_{4 \mathrm{e}}$ :
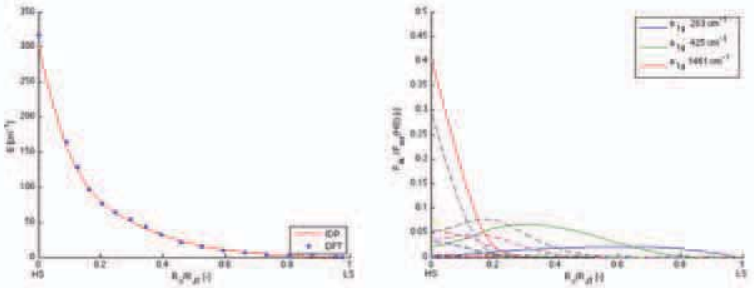

(1) Changes of the energy, IDP modet and DFT results (2) Changes of the forces of the $45 \mathrm{a}_{z}$ modes along the

(e) IDP of the IT $\mathrm{I}_{\mathrm{h}}$ to $\mathrm{C}_{2 \mathrm{n}}\left({ }^{2} \mathrm{~B}_{4}\right)$ distortion in $\mathrm{C}_{\mathrm{ses}}$ :

Figure 3. The multimode analysis of the JT distortion in $\mathrm{C}_{60}^{-}$considering the different pathways. [Color figure can be viewed in the online issue, which is available at wileyonlinelibrary.com.]

\section{Conclusions}

DFT is nowadays the most common theoretical method in quantum chemistry, and it can be successfully applied for a detailed analysis of a range of problems in chemistry. The multideterminantal approach to DFT is an accurate and efficient way for a study of the JT effect, even in the case of relatively large molecule such as the $\mathrm{C}_{60}^{-}$anion, which has a very subtle and complicated distortion. This becomes very important, as multireference wavefunction methods, which are commonly employed for the investigation of the JT effect, are usually not affordable for systems like this one. The DFT based multimode analysis presented in this article, IDP method, answers the question which normal modes, out of all totally symmetric normal modes of the LS minimum energy conformation, are responsible for the changes in geometry and for the $E_{\mathrm{JT}}$, and how their contribution changes along the minimal energy path. Most of the stabilization energy is achieved by the hardest JT active modes, relatively early along the distortion path. The relaxation of the geometry, which yields the energy minimum on the potential energy surface in the final part of the distortion path, is encountered by softer modes. These observations are so far general for all the analyzed molecules with this model. The results obtained by MDDFT and IDP are consistent and in good agreement with other theoretical considerations. Finally, in this work, it was shown once more, that both methods can be considered as reliable tools for better understanding of the JT phenomenon.

\section{Acknowledgments}

The COST-CMTS Action CM1002 "COnvergent Distributed Environment for Computational Spectroscopy (CODECS)" is acknowledged. The authors are very grateful to Prof. Carl-Wilhem Schläpfer for his constant interest and advice.

Keywords: multideterminantal DFT - Jahn-Teller effect - fullerene anion - multimode problem • intrinsic distortion path 
[1] M. Bühl, A. Hirsch, Chem. Rev. 2001, 101, 1153.

[2] (a) C. C. Chancey, M. C. M. O'Brien, The Jahn-Teller Effect in $\mathrm{C}_{60}$ and Other Icosahedral Complexes; Princeton University Press: Princeton, New Jersey, 1997; (b) L. S. Wang, J. Conceicao, R. E. Smalley, Chem. Phys. Lett. 1991, 182, 5; (c) P. J. Fagan, J. C. Calabrese, B. Malone, Acc. Chem. Res. 1992, 25, 134.

[3] M. A. Greaney, S. M. Gorun, J. Phys. Chem. 1991, 95, 7142.

[4] D. Dubois, K. M. Kadish, S. Flanagan, S. E. Haufler, L. P. F. Chibante, L. J. Wilson, J. Am. Chem. Soc. 1991, 113, 4364.

[5] D. Dubois, K. M. Kadish, S. Flanagan, L. J. Wilson, J. Am. Chem. Soc. 1991, 113, 7773 .

[6] Q. Xie, E. Perez-Cordero, L. Echegoyen, J. Am. Chem. Soc. 1992, 114, 3978.

[7] T. T. M. Palstra, R. C. Haddon, Solid State Commun. 1994, 92, 71.

[8] M. J. Rosseinsky, A. P. Ramirez, S. H. Glarum, D. W. Murphy, R. C. Haddon, A. F. Hebard, T. T. M. Palstra, A. R. Kortan, S. M. Zahurak, A. V. Makhija, Phys. Rev. Lett. 1991, 66, 2830.

[9] N. Iwahara, T. Sato, K. Tanaka, L. F. Chibotaru, Phys. Rev. B. 2010, 82, 245409.

[10] W. H. Green, S. M. Gorun, G. Fitzgerald, P. W. Fowler, A. Ceulemans, B. C. Titeca, J. Phys. Chem. 1996, 100, 14892.

[11] N. Koga, K. Morokuma, Chem. Phys. Lett. 1992, 196, 191

[12] I. D. Hands, J. L. Dunn, C. A. Bates, M. J. Hope, S. R. Meech, D. L. Andrews, Phys. Rev. B. 2008, 77, 115445.

[13] O. Gunnarsson, H. Handschuh, P. S. Bechthold, B. Kessler, G. Ganteför, W. Eberhardt, Phys. Rev. Lett. 1995, 74, 1875.

[14] H. A. Jahn, E. Teller, Proc. Roy. Soc. London Ser. A 1937, 161, 220.

[15] I. B. Bersuker, The Jahn-Teller Effect; Cambridge University Press: Cambridge, 2006

[16] I. B. Bersuker, V. Z. Polinger, Vibronic Interactions in Molecules and Crystals; Springer-Verlag: Berlin, 1989

[17] H. Köppel, D. R. Yarkony, H. Barentzen, Eds. The Jahn-Teller Effect, Fundamentals and Implications for Physics and Chemistry: Springer Series in Chemical Physics, Vol. 97; Springer: Heidelberg, Dordrecht, London, New York, 2009.

[18] (a) A. Ceulemans, L. G. Vanquickenborne, Struct. Bond. 1989, 71, 125; (b) A. Ceulemans, D. Beyens, L. G. Vanquickenborne, J. Am. Chem. Soc. 1984, 106, 5824.

[19] (a) J. L. Dunn, C. A. Bates, Phys. Rev. B. 1995, 52, 5996; (b) I. D. Hands, J. L. Dunn, C. A. Bates, Phys. Rev. B. 2006, 73, 235425.

[20] M. C. M. O'Brien, Phys. Rev. B. 1996, 53, 3775.

[21] G. B. Adams, O. F. Sankey, J. B. Page, M. O'Keeffe, Chem. Phys. 1993, $176,61$.

[22] G. Klupp, K. Kamaras, In The Jahn-Teller-Effect Fundamentals and Implications for Physics and Chemistry: Springer Series in Chemical Physics, Vol. 97; H. Köppel, D. R. Yarkoni, H. Barentzen, Eds.; Springer, Heidelberg, Dordrecht, London, New York, 2009; pp. 489-515.

[23] (a) R. Bruyndonckx, C. A. Daul, P. T. Manoharan, E. Deiss, Inorg. Chem. 1997, 36, 4251; (b) T. K. Kundu, R. Bruyndoncks, C. A. Daul, P. T. Manoharan, Inorg. Chem. 1999, 38, 3931; (c) M. L. Zlatar, C.-W. Schläpfer, C. A. Daul, In The Jahn-Teller-Effect Fundamentals and Implications for Physics and Chemistry: Springer Series in Chemical Physics, Vol. 97; $\mathrm{H}$. Köppel, D. R. Yarkoni, H. Barentzen, Eds.; Springer: Heidelberg, Dordrecht, London, New York, 2009; pp. 131-165.

[24] (a) M. Zlatar, C.-W. Schläpfer, E. P. Fowe, C. A. Daul, Pure Appl. Chem. 2009, 81, 1397; (b) M. Zlatar, M. Gruden-Pavlovic, C.-W. Schläpfer, C. A. Daul, J. Mol. Struct. (Theochem) 2010, 954, 86; (c) Gruden-Pavlovic, M.; M. Zlatar, C. W. Schläpfer, C. A. Daul, J. Mol. Struct. (Theochem) 2010, 954, 80; (d) M. Zlatar, M. Gruden-Pavlovic, C.-W. Schläpfer, C. A. Daul, Chimia 2010, 64, 161.
[25] D. Reinen, M. Atanasov, W. Massa, Z. Anorg. Allg. Chem. 2006, 632, 1375.

[26] (a) M. Atanasov, P. Comba, J. Mol. Struct. 2007, 38, 157; (b) M. Atanasov, P. Comba, C. A. Daul, A. Hauser, J. Phys. Chem. A. 2007, 38, 9145; (c) M. T. Barriuso, P. Garcia-Fernandez, J. A. Aramburu, M. Moreno, Int. J. Quantum Chem. 2003, 91, 202.

[27] J. Aramburu, M. Barriuso, P. Garcia-Fernandez, M. Moreno, Adv. Quantum Chem. 2004, 44, 445.

[28] D. Reinen, M. Atanasov, P. Köhler, D. Babel, Coord. Chem. Rev. 2010, 254,2703

[29] M. Zlatar, J.-P. Brog, A. Tschannen, M. Gruden-Pavlovic, C. Daul, In Vibronic Interactions and Jahn-Teller Effect: Theory and Applications, Progress in Theoretical Chemistry and Physics, Vol. 23; M. Atanasov, C. Daul, P. Tregenna-Piggott, Eds.; Springer: Heidelberg, Dordrecht, London, New York, 2012; pp. 25-38.

[30] (a) A. K. Theophilou, J. Phys. C 1979, 12, 5419; (b) A. K. Theophilou, P. Papaconstantinou, Phys. Rev. A 2000, 61, 022502.

[31] (a) E. J. Baerends, T. Ziegler, J. Autschbach, D. Bashford, A. Bérces, F.M. Bickelhaupt, C. Bo, P. M. Boerrigter, L. Cavallo, D. P. Chong, L. Deng, R.M. Dickson, D. E. Ellis, M. van Faassen, L. Fan, T. H. Fischer, C. Fonseca Guerra, A. Ghysels, A. Giammona, S. J. A. van Gisbergen, A. W. Götz, J. A. Groeneveld, O.V. Gritsenko, M. Grüning, S. Gusarov, F. E. Harris, P. van den Hoek, C. R. Jacob, H. Jacobsen, L. Jensen, J. W. Kaminski, G. van Kessel, F. Kootstra, A. Kovalenko, M. V. Krykunov, E. van Lenthe, D. A. McCormack, A. Michalak, M. Mitoraj, J. Neugebauer, V. P. Nicu, L. Noodleman, V. P. Osinga, S. Patchkovskii, P. H. T. Philipsen, D. Post, C. C. Pye, W. Ravenek, J. I. Rodríguez, P. Ros, P. R. T. Schipper, G. Schreckenbach, J. S. Seldenthuis, M. Seth, J. G. Snijders, M. Solà, M. Swart, D. Swerhone, G. te Velde, P. Vernooijs, L. Versluis, L. Visscher, O. Visser, F. Wang, T. A. Wesolowski, E. M. van Wezenbeek, G. Wiesenekker, S. K. Wolff, T. K. Woo, A. L. Yakovlev. ADF2009.01. Available at: https:// www.scm.com, 2009, Accesed on February 2012; (b) C. F. Guerra, J. G. Sniders, G. te Velde, E. J. Baerends, Theor. Chem. Acc. 1998, 99, 391; (c) G. te Velde, F. M. Bickelhaupt, S. J. A. van Gisbergen, C. F. Guerra, E. J. Baerends, J. G. Sniders, T. Ziegler, J. Comput. Chem. 2001, 22, 932.

[32] S. Vosko, L. Wilk, M. Nussair, Can. J. Phys. 1980, 58, 1200.

[33] M. Gruden-Pavlovic, P. Garcia-Fernandez, L. Adjelkovic, C. A. Daul, M. Zlatar, J. Phys, Chem. A. 2011, 115, 10801.

[34] N. Koga, K. Morokuma, Chem. Phys. Lett. 1992, 196, 191.

[35] V. C. Long, E. C. Schundelr, G. B. Adams, J. B. Page, W. Bietsch, I. Bauer, Phys. Rev. B. 2007, 75, 125402.

[36] N. Iwahara, T. Sato, K. Tanaka, L. F. Chibotaru, In: Vibronic Interactions and the Jahn-Teller Effect: Progress in Theoretical Chemistry and Physics, Vol.23; M. Atanasov, C. A. Daul, P. L. W. Tregenna-Piggott, Eds.; Springer: Heidelberg, Dordrecht, London, New York, 2012, pp.245-264.

[37] N. Manini, A. D. Carso, M. Fabrizio, E. Tosatti, Philos. Mag. B. 2001, 81, 793.

[38] I. D. Hands, J. L. Dunn, C. A. Bates, Phys. Rev. B 2001, 63, 245414.

[39] (a) W. Hug, M. Fedorovsky, Theo. Chem. Acc. 2008, 119, 113; (b) M. Fedorovsky, PyVib2: A program for analyzing vibrational motion and vibrational spectra. Available at: http://pyvib2.sourceforce.net, 2007. Accesed on February 2012. 